\title{
The Computational Complexity of Quantified Reciprocals
}

\author{
Jakub Szymanik \\ Institute for Logic, Language and Computation, University of Amsterdam \\ Institute of Philosophy, University of Warsaw \\ j.szymanik@uva.nl
}

\begin{abstract}
We study the computational complexity of reciprocal sentences with quantified antecedents. We observe a computational dichotomy between different interpretations of reciprocity, and shed some light on the status of the so-called Strong Meaning Hypothesis.
\end{abstract}

Key words: reciprocal expressions, computational complexity, generalized quantifiers, Strong Meaning Hypothesis

\section{Introduction}

The English reciprocal expressions each other and one another are common elements of everyday English. Therefore, it is not surprising that they are extensively studied in the formal semantics of natural language (see e.g. [1]). There are two main approaches to reciprocals in the literature. The long trend of analyzing reciprocals as anaphoric noun phrases with the addition of plural semantics culminates in [2]. A different trend - recently represented in [3] — is to analyze reciprocals as polyadic quantifiers.

In this paper we study the computational complexity of reciprocal sentences with quantified antecedents. We put ourselves in the second tradition and treat reciprocal sentences as examples of a natural language semantic construction that can be analyzed in terms of so-called polyadic lifts of simple generalized quantifiers. We propose new relevant lifts and focus on their computational complexity. From this perspective we also investigate the cognitive status of the so-called Strong Meaning Hypothesis proposed in [4].

\subsection{Basic Examples}

We start by recalling examples of reciprocal sentences, versions of which can be found in English corpora (see [4]). Let us first consider the sentences (1)-(3).

\footnotetext{
* I would like to thank Johan van Benthem, Nina Gierasimczuk, Theo Janssen, Marcin Mostowski, Jouko Väänänen, Dag Westerståhl, and Yoad Winter for many comments and inspiration. The author was supported by a Marie Curie Early Stage Research fellowship in the project GloRiClass (MEST-CT-2005-020841).
} 
(1) At least 4 parliament members refer to each other indirectly.

(2) Most Boston pitchers sat alongside each other.

(3) Some Pirates were staring at each other in surprise.

The possible interpretations of reciprocity exhibit a wide range of variations. For example, sentence (1) implies that there is a subset of parliament members of cardinality at least 4 such that each parliament member in that subset refers to some statement of each of the other parliament members in that subset. However, the reciprocals in the sentences (2) and (3) have different meanings. Sentence (2) entails that each pitcher from the set containing most of the pitchers is directly or indirectly in the relation of sitting alongside with each of the other pitchers from that set. Sentence (3) says that there was a group of pirates such that every pirate belonging to the group stared at some other pirate from the group. Typical models satisfying (1)-(3) are illustrated at Figure 1. Following [4] we will call the illustrated reciprocal meanings strong, intermediate, and weak, respectively.
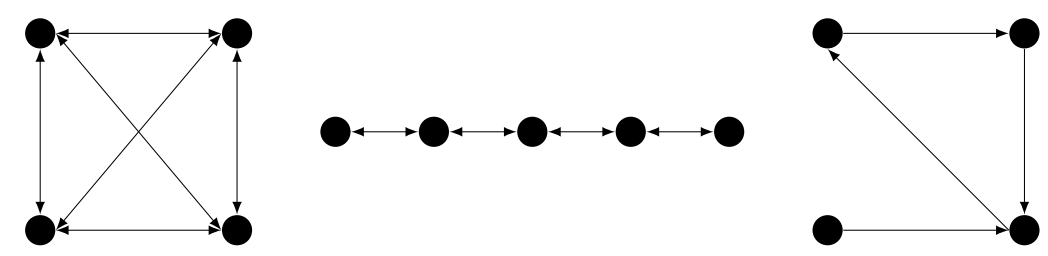

Fig. 1. On the left, a model satisfying sentence (1). This is so-called strong reciprocity. Each element is related to each of the other elements. In the middle, a model satisfying sentence (2) in a context with at most 9 pitchers. This is intermediate reciprocity. Each element in the witness set of the quantifier Most is related to each other element in that set by a chain of relations. On the right, a model satisfying sentence (3), so-called weak reciprocity. For each element there exists a different related element.

In general according to [4] there are 2 parameters characterizing variations of reciprocity. The first one relates to how the scope relation $R$ should cover the domain $A$ (in our case restricted by a quantifier in antecedent). We have 3 possibilities:

FUL Each pair of elements from $A$ participates in $R$ directly.

LIN Each pair of elements from $A$ participates in $R$ directly or indirectly.

TOT Each element in $A$ participates in the relation $R$ with at least one other element.

The second parameter determines whether the relation $R$ between individuals in $A$ is the extension of the reciprocal's scope $(R)$, or is obtained from the extension by ignoring the direction in which the scope relation holds $\left(R^{\vee}=R \cup R^{-1}\right)$.

By combining these 2 parameters we got 6 possible meanings for reciprocals. We encountered already 3 of them: strong reciprocity $(\mathrm{FUL}(R))$, intermediate reciprocity $(\operatorname{LIN}(R))$, and weak reciprocity $(\operatorname{TOT}(R))$. There are 3 new logical possibilities: strong alternative reciprocity $\left(\mathrm{FUL}\left(R^{\vee}\right)\right)$, intermediate alternative 
reciprocity $\left(\operatorname{LIN}\left(R^{\vee}\right)\right)$, and weak alternative reciprocity (TOT $\left.\left(R^{\vee}\right)\right)$. Among alternative reciprocal interpretations two are linguistically attested: intermediate alternative reciprocity exhibited by sentence (4) and weak alternative reciprocity occurring in sentence (5)(See Figure 2).

(4) Most stones are arranged on top of each other.

(5) All planks were stacked atop of each other.
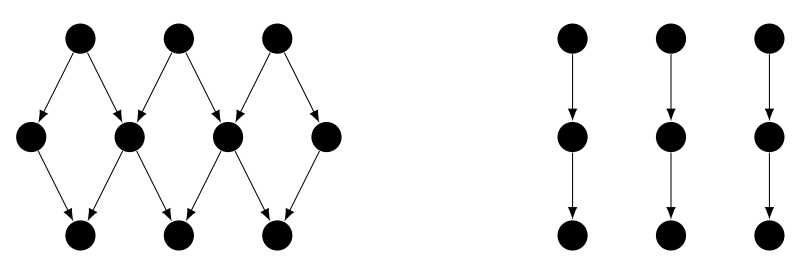

Fig. 2. On the left a model, satisfying sentence (4), so-called intermediate alternative reciprocity. Ignoring the direction of arrows, every element in the witness set of the quantifier Most is connected directly or indirectly. On the right a model satisfying sentence (5), so-called weak alternative reciprocity. Each element participates with some other element in the relation as the first or as the second argument, but not necessarily in both roles.

Noticed also that under certain properties of the relation some of the possible definitions become equivalent. For example, if the relation in question is symmetric, then obviously alternative versions reduce to their "normal" counterparts.

\section{$1.2 \quad$ Strong Meaning Hypothesis}

In an attempt to explain variations in the literal meaning of the reciprocal expressions the so-called Strong Meaning Hypothesis (SMH) was proposed in [4]. According to this principle, the reading associated with the reciprocal in a given sentence is the strongest available reading which is consistent with relevant information supplied by the context. In [3] a considerably simpler system in which reciprocal meanings are derived directly from semantic restrictions using SMH was suggested. Our results show that the various meanings assigned to reciprocals with quantified antecedents differ drastically in their computational complexity. This fact can be treated as suggesting some improvements for the shifts between possible meanings of reciprocal sentences which are predicted by SMH. We elaborate on this possibility in the last section of the paper before we reach the conclusions. 


\section{Reciprocal Expressions as Quantifiers}

\subsection{Generalized Quantifiers}

As this paper analyzes reciprocity in the framework of the theory of generalized quantifiers, we start by recalling the definition of a generalized quantifier (see [5], [6]).

A generalized quantifier $\mathbf{Q}$ of type $\left(n_{1}, \ldots, n_{k}\right)$ is a class of structures of the form $\mathbb{M}=\left(M, R_{1}, \ldots, R_{k}\right)$, where $M$ is the universe and $R_{i}$ is a subset of $M^{n_{i}}$. Additionally, $Q$ is closed under isomorphism. Syntactically a quantifier $Q$ of type $\left(n_{1}, \ldots, n_{k}\right)$ binds $m=n_{1}+\ldots+n_{k}$ first-order variables, and $k$ formulae. If for all $i: n_{i} \leq 1$, then we say that quantifier is monadic, otherwise we call it polyadic.

In other words, $Q$ is a functional relation associating with each model $\mathbb{M}$ a relation between relations on $M$. Hence, if we fix a model $\mathbb{M}$ we have the following equivalence:

$$
\left(M, R_{1}{ }^{M}, \ldots, R_{k}{ }^{M}\right) \in \mathrm{Q} \Longleftrightarrow \mathrm{Q}_{M}\left(R_{1}, \ldots, R_{k}\right) \text {, where } R_{i}{ }^{M} \subseteq M^{n_{i}} .
$$

As an example consider the quantifier Most of type $(1,1)$. It corresponds to the following class of finite models:

$$
\text { Most }=\left\{\left(M, A^{M}, B^{M}\right): \operatorname{card}\left(A^{M} \cap B^{M}\right)>\operatorname{card}\left(A^{M}-B^{M}\right)\right\} .
$$

In a given model $\mathbb{M}$ the statement $\operatorname{Most}_{M}(A, B)$ says that $\operatorname{card}\left(A^{M} \cap B^{M}\right)>$ $\operatorname{card}\left(A^{M}-B^{M}\right)$.

Generalized quantifiers were succesfully introduced to linguistics in [7].

\subsection{Reciprocals as Polyadic Quantifiers}

Monadic generalized quantifiers provide the most straightforward way to define the semantics of noun phrases in natural language (see [8]). Sentences with reciprocal expressions transform such monadic quantifiers into polyadic ones. We will analyze reciprocal expressions in that spirit by defining appropriate lifts on monadic quantifiers. These lifts are definable in existential second-order logic. For the sake of simplicity we will restrict ourselves to reciprocal sentences with right monotone increasing quantifiers in antecedents. We say that a quantifier $\mathrm{Q}$ is monotone increasing in its right argument, if $Q_{M}(A, B)$ and $B \subseteq B^{\prime} \subseteq M$, then $Q_{M}\left(A, B^{\prime}\right)$. Below defined lifts can be extended to cover also sentences with decreasing and non-monotone quantifiers, for example by following the strategy of bounded composition in [4] or using determiner fitting operator proposed in [9].

In order to define the meaning of the strong reciprocity we make use of the well-know operation on quantifiers called Ramseyfication. Let $Q$ be a right monotone increasing quantifier of type $(1,1)$, we define:

$$
\operatorname{Ram}_{\mathrm{S}}(\mathrm{Q})(A, R) \Longleftrightarrow \exists X \subseteq A[\mathrm{Q}(A, X) \wedge \forall x, y \in X(x \neq y \Rightarrow R(x, y))] .
$$


The result of such a lift is called a Ramsey quantifier. In the same way we can also easily account for the alternative strong reciprocity:

$$
\operatorname{Ram}_{\mathrm{S}} \vee(\mathrm{Q})(A, R) \Longleftrightarrow \exists X \subseteq A[\mathrm{Q}(A, X) \wedge \forall x, y \in X(x \neq y \Rightarrow(R(x, y) \vee R(y, x)))] .
$$

Ram $_{\mathrm{S}}$ is defined analogously for unary quantifiers as for type $(1,1)$, just replace condition $\mathrm{Q}(A, X)$ by $\mathrm{Q}(X)$ in the definition.

In an analogous way we define other lifts to express intermediate, and weak reciprocity and their alternative versions. For intermediate reciprocity we have the following:

$$
\begin{array}{r}
\operatorname{Ram}_{\mathrm{I}}(\mathrm{Q})(A, R) \Longleftrightarrow \exists X \subseteq A[\mathrm{Q}(A, X) \wedge \forall x, y \in X \\
\left(x \neq y \Rightarrow \exists \text { sequence } z_{1}, \ldots, z_{\ell} \in X\right. \text { such that } \\
\left.\left.z_{1}=x \wedge R\left(z_{1}, z_{2}\right) \wedge \ldots \wedge R\left(z_{\ell-1}, z_{\ell}\right) \wedge z_{\ell}=y\right)\right] .
\end{array}
$$

The alternative version is defined naturally. In other words these conditions guarantee that $X$ is connected with respect to $R$ or $R^{\vee}$. Anyway, graph connectedness is not elementary expressible, we need a universal monadic second-order formula. Hence from the definability point of view $\operatorname{Ram}_{I}\left(\operatorname{Ram}_{\mid}{ }^{\vee}\right)$ seems more complicated than $\operatorname{Ram}_{\mathrm{S}}\left(\operatorname{Ram}_{\mathrm{S}}{ }^{\vee}\right)$. However, as we will see in the next chapter of this paper it is not always the case when a computational complexity perspective is taken into account.

For weak reciprocity we take the following lifts and its alternative version:

$$
\operatorname{Ram}_{\mathrm{W}}(\mathrm{Q})(A, R) \Longleftrightarrow \exists X \subseteq A[\mathrm{Q}(A, X) \wedge \forall x \in X \exists y \in X(x \neq y \wedge R(x, y))] .
$$

All these lifts produce polyadic quantifiers of type $(1,2)$. We will call the values of these lifts (alternative) strong, (alternative) intermediate and (alternative) weak reciprocity, respectively.

The linguistic application of these lifts is straightforward. For example, formulae (6)-(10) give the readings to sentences (1)-(5).

(6) $\operatorname{Ram}_{\mathrm{S}}$ (At least 4)(MP, Refer-indirectly).

(7) $\operatorname{Ram}_{\mathrm{I}}$ (Most)(Pitcher, Sit-next-to).

(8) $\operatorname{Ram}_{W}$ (Some)(Pirate, Staring-at).

(9) $\operatorname{Ram}_{1} \vee$ (Most)(Stones, Arranged-on-top-of).

(10) $\operatorname{Ram}_{W}{ }^{\vee}($ All) (Planks, Stack-atop-of).

\subsection{The Computational Complexity of Quantifiers}

By the complexity of a quantifier $Q$ we mean the computational complexity (see e.g. [10]) of the corresponding class of finite models. For example, consider a quantifier $\mathrm{Q}$ of type $(1,2)$. In that case $\mathrm{Q}$ is a class of finite models of the following form $\mathbb{M}=\left(M, A^{M}, R^{M}\right)$. We are now given a model $\mathbb{M}$ of that form and a quantifier $\mathrm{Q}$. We can assume that the universe $M$ consists of natural numbers: $M=\{1, \ldots, m\}, A^{M}$ is a subset of $M$ and $R^{M}$ is a binary relation 
over $M$. Our computational problem is to decide whether $\mathbb{M} \in \mathbf{Q}$. Equivalently, does $\mathbb{M} \models \mathrm{Q}(A, R)$ ?

Generalized quantifiers in finite models - from the point of view of computational complexity - were considered for the first time in [11], where the following terminology was introduced. We say that a quantifier $Q$ is $N P$-hard if the corresponding class of finite models is NP-hard. Q is mighty (NP-complete) if the corresponding class belongs to NP and is NP-hard.

It was observed in [12] that some natural language quantifiers when assuming their branching interpretation are mighty. More results of this type can be found in [13]. Essentially all of the proofs of NP-completeness for branching quantifiers are based on a kind of Ramsey property which is expressible by means of branching. The main application of branching quantifiers in linguistics is within the study of sentences like:

(11) Some relative of each villager and some relative of each townsman hate each other.

(12) Most villagers and most townsmen hate each other.

However, all these NP-complete natural language constructions are ambiguous. Their reading varies between easy and difficult interpretations. Moreover, such sentences can hardly be found in natural language corpora (see [13]). One of the goals of this paper is to present mighty natural language quantifiers which not only occur frequently in everyday English but are also one of the sources of its complexity.

\section{Complexity of the Ramseyfication}

We will restrict ourselves to finite models. We identify models of the form $\mathbb{M}=$ $\left(M, A^{M}, R^{M}\right)$, where $A^{M} \subseteq M$ and $R^{M} \subseteq M^{2}$, with colored graphs. Remember that we are considering only monotone increasing quantifiers. Hence, in graphtheoretical terms we can say that $\mathbb{M}=\operatorname{Ram}_{\mathrm{S}}(\mathrm{Q})(A, R)$ if and only if there is a complete subgraph in $A^{M}$ with respect to $R^{M}$ of a size bounded below by the quantifier Q. $R^{M}$ is the extension of a reciprocal relation $R$. If $R$ is symmetric then we are obviously dealing with undirected graphs. In such cases Rams and $\operatorname{Ram}_{\mathrm{S}}{ }^{\vee}$ are equivalent. Otherwise, if the reciprocal relation $R$ is not symmetric, our models become directed graphs. In the following two subsections we will restrict ourselves to undirected graphs and prove that some strong reciprocal quantified sentences are then NP-complete. As undirected graphs are special case of directed graphs then general problems for them also have to be NP-complete.

\subsection{Simple Observations}

Counting Quantifiers To decide whether in some model $\mathbb{M}$ sentence $\operatorname{Ram}_{\mathrm{S}}$ (At least $\left.\mathrm{k}\right)(A, R)$ is true we have to solve the CLIQUE problem for $M$ and $k$. A brute force algorithm to find a clique in a graph is to examine each subgraph with at least $k$ vertices and check if it forms a clique. This means that 
for every fixed $k$ the computational complexity of $\operatorname{Ram}_{\mathrm{S}}$ (At least $\mathbf{k}$ ) is in PTIME. For instance, $\operatorname{Ram}_{\mathrm{S}}$ (At least 5 ) is computable in a polynomial time. Moreover, notice that the strong reciprocal sentence $\operatorname{Ram}_{\mathrm{S}}\left(\exists^{\geq k}\right)(A, R)$ is equivalent to the following first-order formula:

$$
\exists x_{1} \ldots \exists x_{k}\left[\bigwedge_{1 \leq i<j \leq k} x_{i} \neq x_{j} \wedge \bigwedge_{1 \leq i \leq k} A\left(x_{i}\right) \wedge \bigwedge_{\substack{1 \leq i \leq k \\ 1 \leq j \leq k}} R\left(x_{i}, x_{j}\right)\right] .
$$

However, when we consider natural language semantics from a procedural point of view it is natural to assume that people rather have one quantifier concept At least $k$, for every natural number $k$, than the infinite set of concepts At least 1 , At least $2, \ldots$. It seems reasonable to suppose that we learn one mental algorithm to understand each of the counting quantifiers At least $k$, At most $\mathrm{k}$, and Exactly $\mathrm{k}$, no matter which natural number $k$ actually is. Mathematically we can account for this idea by introducing counting quantifiers. The counting quantifier $C \geq A$ says that the number of elements satisfying some property in a model $\mathbb{M}$ is greater or equal to the cardinality of set $A \subseteq M$. In other words, the idea here is that determiners like At least $k$ express a relation between number of elements satisfying certain property and cardinality of some prototypical set $A$. For instance, determiner At least $k$ corresponds to the quantifier $C \geq A$ such that $\operatorname{card}(A)=k$. Therefore, determiners At least 1, At least 2, At least 3, ... are interpreted by one counting quantifier $\mathrm{C} \geq \mathrm{A}$ - just set $A$ has to be chosen differently in every case. ${ }^{1}$

The quantifier $\operatorname{Ram}_{S}\left(C^{\geq A}\right)$ expresses the general schema for reciprocal sentences with counting quantifiers in antecedents. Such general pattern defines NP-complete problems

Proposition 1 The quantifier $\operatorname{Ram}_{\mathrm{S}}(\mathrm{C} \geq \mathrm{A})$ is mighty.

Proportional Quantifiers We can give one more general example of strong reciprocal sentences which are NP-complete. Let us consider the following sentences:

(13) Most members of the parliament refer to each other indirectly.

(14) At least one third of the members of the parliament refer to each other indirectly.

(15) At least $q \times 100 \%$ of the members of the parliament refer to each other indirectly.

We will call these sentences the strong reciprocal sentences with proportional quantifiers. Their general form is given by the sentence schema (15), where $q$ can be interpreted as any rational number between 0 and 1 . These sentences say

\footnotetext{
${ }_{1}$ Alternatively we can introduce two-sorted variants of finite structures, augmented by a infinite number sort. Then we can define counting quantifiers in a way that the numeric constants in a quantifier refer to the number domain (see e.g. [14], [15]).
} 
that there is a clique, $C l \subseteq A$, where $A$ is the set of all parliament members, such that $\operatorname{card}(C l) \geq q \times \operatorname{card}(A)$.

For any rational number $q$ between 0 and 1 we say that a set $A \subseteq U$ is $q$-big if and only if $\frac{\operatorname{card}(A)}{\operatorname{card}(U)} \geq q$. In this sense $q$ determines a proportional quantifier $\mathrm{R}_{\mathrm{q}}$

of type $(1,1)$ such that $\mathbb{M} \models \mathrm{R}_{\mathrm{q}}(A, B)$ iff $\frac{\operatorname{card}\left(A^{M}\right)}{\operatorname{card}\left(B^{M}\right)} \geq q$. The strong reciprocal lift of this quantifier, $\operatorname{Ram}_{\mathrm{S}}\left(\mathrm{R}_{\mathrm{q}}\right)$, is of type $(1,2)$ and might be used to express meanings of sentences like (13)-(15). We will call the quantifiers of the form $\operatorname{Ram}_{\mathrm{S}}\left(\mathrm{R}_{\mathrm{q}}\right)$ proportional Ramsey quantifiers. In [16] the following was observed:

Proposition 2 If $q$ is a rational number between 0 and 1, then the quantifiers $\operatorname{Ram}_{\mathrm{S}}\left(\mathrm{R}_{\mathrm{q}}\right)$ is mighty.

\subsection{General Dichotomy}

Our examples show that the strong interpretation of some reciprocal sentences is NP-complete. In this section we will describe a class of unary monadic quantifiers for which the strong reciprocal interpretation is PTIME computable.

Following [17] we will identify monotone simple unary quantifiers with number-theoretic functions, $f: \omega \rightarrow \omega$, such that for all $n \in \omega, f(n) \leq n+1$. In that setting the quantifier $\mathrm{Q}_{f}$ (corresponding to $f$ ) says of a set $A$ that it has at least $f(n)$ elements, where $n$ is the cardinality of the universe. Therefore, given $f: \omega \rightarrow \omega$, we define:

$$
\left(\mathrm{Q}_{f}\right)_{M}(A) \Longleftrightarrow \operatorname{card}(A) \geq f(\operatorname{card}(M))
$$

Our crucial notion goes back to the paper [18]. We say that a function $f$ (quantifier $\mathrm{Q}_{f}$ ) is bounded if

$$
\exists m \forall n(f(n)<m \vee n-m<f(n)) .
$$

Otherwise $f$ and the corresponding $Q_{f}$ are unbounded. Typical bounded functions are: $f(n)=1$ (corresponding to $\exists$ ) and $f(n)=n$ (corresponding to $\forall$ ). The first one is bounded from above by 2 as for every $n$ we have $f(n)=1<2$. The second one is bounded below by 1 , for every $n, n-1<n$. Unbounded functions are for example: $\left\lceil\frac{n}{2}\right\rceil,\lceil\sqrt{n}\rceil,\lceil\log n\rceil$, where $\lceil p\rceil$ is the ceiling function of $p$. We illustrate the situation in the Figure 3.

In what follows we will show that the PTIME computable bounded quantifiers of type (1) are closed on the strong reciprocal lift.

Proposition 3 If a monotone increasing quantifier $\mathrm{Q}_{f}$ is PTIME computable and bounded, then the reciprocal quantifier $\operatorname{Ram}_{\mathrm{S}}\left(\mathrm{Q}_{f}\right)$ is PTIME computable.

Proof. Assume that $f$ is PTIME computable and bounded. Then there exists a number $m$ such that for every $n$ the following disjunction holds $(f(n)<m$ or $n-m<f(n))$.

Let us fix a graph model $\mathbb{G}=(V, E)$, where $\operatorname{card}(V)=n$. 


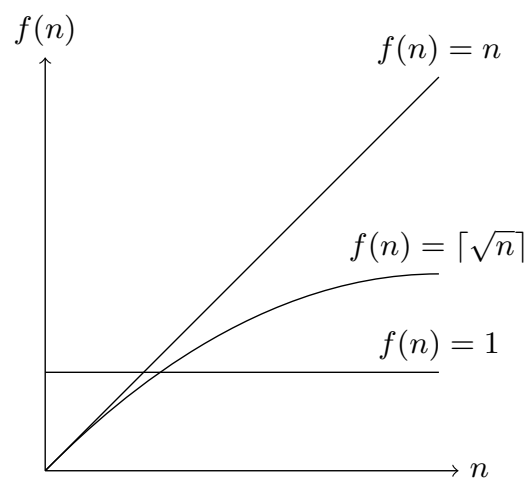

Fig. 3. The functions $f(n)=1$ and $f(n)=n$ are bounded. The function $\lceil\sqrt{n}\rceil$ is unbounded.

Assume that $f(n)<m$. First observe that if there exists a clique of size greater than $f(n)$ then there has to be also a clique of size exactly $f(n)$. Thus to decide whether $\mathbb{G} \in \mathrm{R}_{\mathrm{f}}$ it is enough to check if there is a clique of size $f(n)$ in $\mathbb{G}$. We know that $f(n)<m$. Hence we only need to examine all subgraphs up to $m-1$ vertices. For each of them we can check in a polynomial time whether it forms a clique. Moreover, the number of all subgraphs of size $\leq m-1$ in the graph $\mathbb{G}$ is

$$
\left(\begin{array}{c}
n \\
m-1
\end{array}\right)<n^{m-1} .
$$

Therefore, the whole procedure is bounded by a polynomial of fixed degree.

Let us consider the second case. Notice that as the PTIME class is closed under taking complements it suffices to show a polynomial-time procedure for detecting that there is no clique of size $f(n)$. If $n-m<f(n)$, then $n-f(n)+1 \leq m$. Therefore, the procedure which goes through all subgraphs up to size $n-f(n)+1$ is bounded by $n^{m}$ and hence can be also computed in polynomial time. It is enough to justify that such procedure does the job. To see it observe the following: if there is no clique of size up to $n-f(n)+1$, then there is no clique bigger or equal to $f(n)$. This fact follows simply by considering two cases. First, if $f(n) \geq n-f(n)+1$, then there cannot be clique of size $\geq f(n)$ without clique of size $n-f(n)+1$. Otherwise, if $f(n)<n-f(n)+1$ then checking all subgraphs up to $n-f(n)+1$-elements we will also examine all subgraphs of size $f(n)$.

Therefore, in every case when $f$ is bounded and computable in a polynomial time we simply run two above algorithms for every possible case. This modelchecking procedure for $\mathrm{R}_{\mathrm{f}}$ simply tests clique property on all subgraphs up to $m$ elements, where $m$ is fixed and independent from the size of a universe. Therefore, it is bounded by a polynomial. 
It does not matter whether we consider undirected or directed graphs as in both cases checking whether a given subgraph is complete can be done in polynomial time. It also does not make any difference whether we are interested in being complete subgraph with respect to $R$ or $R^{\vee}$, so the result holds also for $\operatorname{Ram}_{\mathrm{S}}{ }^{\vee}\left(Q_{f}\right)$.

Moreover, notice that the relativization $\mathrm{Q}_{f}^{\text {rel }}$ of $\mathrm{Q}_{f}$ is the right monotone type $(1,1)$ quantifier: $\left(Q_{f}^{r e l}\right)_{M}(A, B) \Longleftrightarrow \operatorname{card}(A \cap B) \geq f(\operatorname{card}(A))$. Thus, the restriction is not essential and the result may be easily translated for type $(1,1)$ quantifiers.

Notice that the property of boundness plays also a crucial role in the definability theory of polyadic lifts. In [18] it has been shown that the Ramseyfication of $\mathrm{Q}$ is definable in $F O(\mathrm{Q})$ if and only if $\mathrm{Q}$ is bounded. They also obtained similar results for branching and resumption (see [18]).

\section{Complexity of the Intermediate and Weak Lifts}

Analogously to the case of strong reciprocity we can also express the meanings of intermediate and weak reciprocal lifts in graph-theoretical terms. We say that $\mathbb{M} \models \operatorname{Ram}_{\mathrm{I}}(\mathrm{Q})(A, R)$ if and only if there is a connected subgraph in $A^{M}$ of a size bounded from below by the quantifier $\mathrm{Q} . \mathbb{M} \models \operatorname{Ram}_{\mathrm{W}}(\mathrm{Q})(A, R)$ if and only if there is a subgraph in $A^{M}$ of a proper size without isolated vertices. All with respect to the relation $R^{M}$, either symmetric or asymmetric.

We prove that the class of PTIME quantifiers is closed under the (alternative) intermediate lift and the (alternative) weak lift.

Proposition 4 If a monotone increasing quantifier $\mathrm{Q}$ is a PTIME computable quantifier, then the quantifiers $\operatorname{Ram}_{\mathrm{I}}(\mathrm{Q})$ and $\operatorname{Ram}_{1}{ }^{\vee}(\mathrm{Q})$ are PTIME computable.

Proof. We consider only the case of the quantifier $\operatorname{Ram}_{\mathrm{I}}(\mathrm{Q})$, the proof for the alternative case is analogous. Let $\mathbb{G}=(V, A, E)$ be a directed colored graphmodel. To check whether $\mathbb{G} \in \operatorname{Ram}_{1}(\mathrm{Q})$ compute all connected components of the subgraph determined by $A$. For example, you can use a breadth-first search algorithm that begins at some node and explores all the connected neighboring vertices. Then for each of those nearest nodes, it explores their unexplored connected neighbor vertices, and so on, until it finds the full connected subgraph. Next, it chooses a node which does not belong to this subgraph and starts searching for the connected subgraph containing it. Since in the worst case this breadth-first search has to go through all paths to all possible vertices, the time complexity of the breadth-first search on the whole $\mathbb{G}$ is $O(\operatorname{card}(V)+\operatorname{card}(E))$. Moreover, the number of the components in $A$ is bounded by $\operatorname{card}(A)$. Having all connected components it is enough to check whether there is a component $C$ of a proper size, i.e. does $\mathrm{Q}(A, C)$ hold for some connected component $C$. This can be checked in a polynomial time as $Q$ is PTIME computable quantifier. Hence, $\operatorname{Ram}_{l}(\mathrm{Q})$ is in PTIME.

The next proposition follows immediately. 
Proposition 5 If a monotone increasing quantifier $\mathrm{Q}$ is a PTIME computable quantifier, then the quantifiers $\operatorname{Ram}_{\mathrm{W}}(\mathrm{Q})$ and $\operatorname{Ram}_{\mathrm{W}}{ }^{\vee}(\mathrm{Q})$ are PTIME computable.

These results show that the intermediate and the weak reciprocal lifts do not increase the complexity of quantifier sentences in such a drastic way as may happen in the case of strong reciprocal lifts. In other words, in many contexts intermediate and weak interpretations are relatively easy as opposed to the strong reciprocal reading.

\section{A Complexity Perspective on SMH}

The Strong Meaning Hypothesis proposed in [4] to predict the proper reading of sentences containing reciprocal expressions. According to SMH the reciprocal expression is interpreted as having logically the strongest truth conditions that are consistent with a given context. Therefore, if it is only consistent with specified facts, then a statement containing each other will be interpreted as a strong reciprocal sentence. Otherwise, an interpretation will shift toward the logically weaker intermediate or weak readings, depending on a context.

$\mathrm{SMH}$ is quite an effective pragmatic principle. We will discuss the shifts $\mathrm{SMH}$ predicts from the computational complexity point of view referring to the results provided in the previous sections.

Let us first think about the meaning of a sentence in the intensional way - identifying the meaning of an expression with an algorithm recognizing its denotation in a finite model ${ }^{2}$. Such algorithms can be described by investigating how language users evaluate the truth-value of sentences in various situations. On the cognitive level it means that subjects have to be equipped with mental devices to deal with meanings of expressions. Moreover, it is cognitively plausible to assume that we have one mental device to deal with most instances of the same semantic construction. For example, we believe that there is one mental algorithm to deal with the counting quantifier, At least $k$, in most possible contexts, no matter what natural number $k$ is. Thus, in the case of logical expressions, like quantifiers, the analogy between meanings and algorithms seems uncontroversial.

However, notice that some sentences, being intractable, are too hard for identifying their truth-value directly by investigating a model. The experience in writing programs suggests that we can claim a sentence to be difficult when it cannot be computed in a polynomial time. Despite the fact that some sen-

\footnotetext{
${ }^{2}$ This approach — going back to [19] — exists in the linguistic literature in the different levels of transparency (see e.g.[20]).
} 
tences are sometimes ${ }^{3}$ too hard for comprehension, we can find their inferential relations with relatively easier sentences.

According to SMH any reciprocal sentence, if it is possible, should be interpreted as a strong reciprocal sentence. We have shown that the strong interpretation of sentences with quantified antecedents is sometimes intractable but intermediate and weak reading are always easy to comprehend. In other words, it is reasonable to suspect that in some linguistic situations the strong reciprocal interpretation is cognitively much more difficult than the intermediate or the weak interpretation. This prediction makes sense under the assumption that $P \neq N P$ and that human mind is bounded by computational restrictions. We omit a discussion here and only recall that computational restrictions for cognitive abilities are widely treated in the literature. For example, in philosophy [21], study of reasoning [22], cognitive science [23], linguistics [24], and formal semantics [25]. In [26] the so-called P-Cognition Thesis was explicitly formulated: P-Cognition Thesis Human cognitive (linguistic) capacities are constrained by polynomial-time computability.

What happens if subject is supposed to deal with a sentence too hard for a direct comprehension. One possibility is that the subject will try to establish the truth-value of a sentence indirectly, by shifting to an accessible inferential meaning. That will be - depending on the context - the intermediate or the weak interpretation - both being implied by the strong interpretation.

Summing up, our descriptive complexity perspective on reciprocity shows that it might be not always possible to interpret a reciprocal sentence in the strong way as SMH suggests. If a sentence in question would be intractable under the strong reciprocal interpretation then people will turn to tractable readings, like intermediate and weak. Our observations give a cognitively reasonable argument for some shifts to occur, even though they are not predicted by SMH. For example, SMH assumes that the following sentence should be interpreted as the strong reciprocal statement.

(16) Most parliament members refer to each other indirectly.

However, we know that this sentence is NP-complete. Therefore, if the set of parliament members is big enough then the statement is intractable under the strong interpretation. It gives a perfect reason to switch for a weaker interpretations.

\section{Conclusion}

By investigating reciprocal expressions in the computational paradigm we found the differences in the complexity between various interpretations of reciprocal

\footnotetext{
3 That the general problem is hard does not show that all instances normally encountered are hard. It is the matter of empirical studies to provide us with data about computational complexity influence on our everyday linguistic experience. However, we believe that it is reasonable to expect that this happens at least in some situations.
} 
sentences with quantified antecedents. In particular, we proved that for PTIME computable quantifiers the intermediate and the weak reciprocal interpretations are PTIME computable. Moreover, if we additionally assume that a quantifier is bounded then also the strong reciprocal interpretation stays in PTIME. Therefore, the semantic distinctions from [4] seem also solid from a computational perspective. Moreover, identifying meanings with algorithms those results allow us to argue in favor of Strong Meaning Hypothesis.

Many questions arise which are to be answered in future work. Here we will mention only a few of them:

(1) Among the reciprocal sentences we found NP-complete constructions. For example, we have shown that the strong reciprocal interpretations of proportional quantifiers are NP-complete. On the other side, we also proved that the strong reciprocal interpretations of bounded quantifiers are in PTIME. We would like to know where is the precise border between those quantifiers for which Ramseyfication is in PTIME and those for which it is NP-complete. Is it the case that for every function $f$ from some class we have a duality theorem, i.e., $\operatorname{Ram}_{\mathrm{S}}\left(\mathrm{Q}_{f}\right)$ is either PTIME computable or NP-complete? Can we prove under some complexity assumptions that PTIME Ramsey quantifiers are exactly bounded Ramsey quantifiers?

(2) There is a vast literature on the definability of polyadic lifts of generalized quantifiers (see e.g. [18]). We introduced some new linguistically relevant lifts, the weak and the intermediate reciprocal lifts. The next step is to study their definability. For example, we would like to know how the definability questions for $\operatorname{Ram}_{\mathrm{S}}\left(\mathrm{Q}_{f}\right), \operatorname{Ram}_{\mathrm{I}}\left(\mathrm{Q}_{f}\right)$, and $\operatorname{Ram}_{\mathrm{W}}\left(\mathrm{Q}_{f}\right)$ depend on the properties of $f$ ? Another interesting point is to link our operators with other polyadic lifts, like branching.

(3) What about different complexity measures? For example, how one can repeat our story invoking parametrized or average-case complexity?

(4) Is it well enough justified to identify meanings with algorithms? Broader philosophical discussion on applications of complexity theory to natural language semantics seems inevitable.

(5) Finally, we need to investigate the interplay between cognitive difficulty and computational complexity in more detail. Do the differences in the computational complexity really play an important role in the natural language processing as some neuroimaging data suggests (see [27], [28])? For example, we could empirically compare the differences in shifts from the strong interpretation of reciprocal sentences with bounded and proportional quantifiers in antecedents. Our approach predicts that subjects will shift to easier interpretations more frequently in the case of sentences with proportional quantifiers. 


\section{References}

1. Heim, I., Lasnik, H., May, R.: Reciprocity and plurality. Linguistic Inquiry 22 (1991) 63-101

2. Beck, S.: The semantics of different: Comparison operator and relational adjective. Linguistics and Philosophy 23 (2000) 101-139

3. Sabato, S., Winter, Y.: From semantic restrictions to reciprocal meanings. In: Proceedings of FG-MOL 2005, CSLI Publications (2005)

4. Dalrymple, M., Kanazawa, M., Kim, Y., Mchombo, S., Peters, S.: Reciprocal expressions and the concept of reciprocity. Linguistics and Philosophy 21 (1998) $159-210$

5. Mostowski, A.: On a generalization of quantifiers. Fundamenta Mathematicae 44 (1957) 12-36

6. Lindström, P.: First order predicate logic with generalized quantifiers. Theoria 32 (1966) 186-195

7. Barwise, J., Cooper, R.: Generalized quantifiers and natural language. Linguistics and Philosophy 4 (1981) 159-219

8. Peters, S., Westerståhl, D.: Quantifiers in Language and Logic. Clarendon Press, Oxford (2006)

9. Winter, Y.: Flexibility principles in Boolean semantics. The MIT Press, London (2001)

10. Papadimitriou, C.H.: Computational Complexity. Addison Wesley (1993)

11. Blass, A., Gurevich, Y.: Henkin quantifiers and complete problems. Annals of Pure and Applied Logic 32 (1986) 1-16

12. Mostowski, M., Wojtyniak, D.: Computational complexity of the semantics of some natural language constructions. Annals of Pure and Applied Logic 127 (2004) 219227

13. Sevenster, M.: Branches of imperfect information: logic, games, and computation. PhD thesis, Universiteit van Amsterdam (2006)

14. Otto, M.: Bounded Variable Logics and Counting. A study in Finite Models. Volume 9. Springer-Verlag (1997)

15. Grädel, E., Gurevich, Y.: Metafinite model theory. Information and Computation 140 (1998) 26-81

16. Mostowski, M., Szymanik, J.: Computational complexity of some Ramsey quantifiers in finite models. The Bulletin of Symbolic Logic 13 (2007) 281-282

17. Väänänen, J.: Unary quantifiers on finite models. Journal of Logic, Language and Information 6 (1997) 275-304

18. Hella, L., Väänänen, J., Westerståhl, D.: Definability of polyadic lifts of generalized quantifiers. Journal of Logic, Language and Information 6 (1997) 305-335

19. Frege, G.: Über Sinn und Bedeutung. Zeitschrift für Philosophie und philosophische Kritik 100 (1892) 25-50

20. Moschovakis, Y.: A logical calculus of meaning and synonymy. Linguistics and Philosophy 29 (2006) 27-89

21. Cherniak, C.: Minimal rationality. Mind 90 (1981) 161-183

22. Levesque, H.J.: Logic and the complexity of reasoning. Journal of Philosophical Logic 17 (1988) 355-389

23. van Rooij, I.: The tractable cognition thesis. Cognitive Science: A Multidisciplinary Journal 32 (2008) 939-984

24. Ristad, E.S.: The Language Complexity Game (Artificial Intelligence). The MIT Press (1993) 
25. Mostowski, M., Szymanik, J.: Semantical bounds for everyday language. Technical Report ILLC Preprint Series, PP-2006-40, Institute for Logic, Language and Computation, University of Amsterdam (2005) To appear in Semiotica.

26. Frixione, M.: Tractable competence. Minds and Machines 11 (2001) 379-397

27. Mcmillan, C.T., Clark, R., Moore, P., Devita, C., Grossman, M.: Neural basis for generalized quantifiers comprehension. Neuropsychologia 43 (2005) 1729-1737

28. Szymanik, J.: A comment on a neuroimaging study of natural language quantifier comprehension. Neuropsychologia 45 (2007) 2158-2160 